\title{
Far Lateral Craniotomy for Resection of Foramen Magnum Meningioma
}

\author{
Robert T. Wicks ${ }^{1} \quad$ Xiaochun Zhao $^{1} \quad$ Celene B. Mulholland ${ }^{1}$ Peter Nakaji \\ 1 Department of Neurosurgery, Barrow Neurological Institute, \\ St. Joseph's Hospital and Medical Center, Phoenix, Arizona, \\ United States \\ Address for correspondence Peter Nakaji, MD, Department of \\ Neurosurgery, Barrow Neurological Institute, St. Joseph's Hospital \\ and Medical Center, 350 West, Thomas Road; Phoenix, AZ 85013, \\ J Neurol Surg B 2019;80(suppl S4):S355-\$357. \\ United States (e-mail: Neuropub@barrowneuro.org).
}

\begin{abstract}
Keywords

- far lateral

- foramen magnum

- occipital condyle

Objective Foramen magnum meningiomas present a formidable challenge to resection due to frequent involvement of the lower cranial nerves and vertebrobasilar circulation. The video shows the use of a far lateral craniotomy to resect a foramen magnum meningioma.

Design, Setting, and Participant A 49-year-old woman presented with neck pain and was found to have a large foramen magnum meningioma ( - Fig. 1A, B). Drilling of the posterior occipital condyle was required to gain access to the lateral aspect of the brain stem. The amount of occipital condyle resection varies by patient and pathology.

Outcome/Result Maximal total resection of the tumor was achieved ( Fig. 1B, C), and the patient was discharged on postoperative day 4 with no neurologic deficits. The technique for tumor microdissection (-Fig. 2) is shown in the video.

Conclusion Given the close proximity of foramen magnum meningiomas to vital structures at the craniocervical junction, surgical resection with careful microdissection and preservation of the overlying dura to prevent postoperative pseudomeningocele is necessary to successfully manage this pathology in those patients who are surgical candidates.

The link to the video can be found at: https://youtu.be/Mds9N1x2zE0.
\end{abstract}

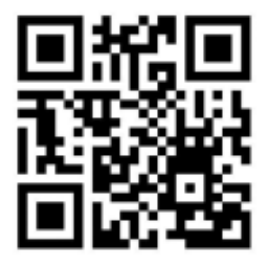

received

April 1, 2019

accepted

August 25, 2019

published online

October 22, 2019 www.thieme.com/skullbasevideos

www.thieme.com/jnlsbvideos (c) 2019 Georg Thieme Verlag KG Stuttgart · New York

License terms

10.1055/s-0039-1698828. ISSN 2193-6331. 

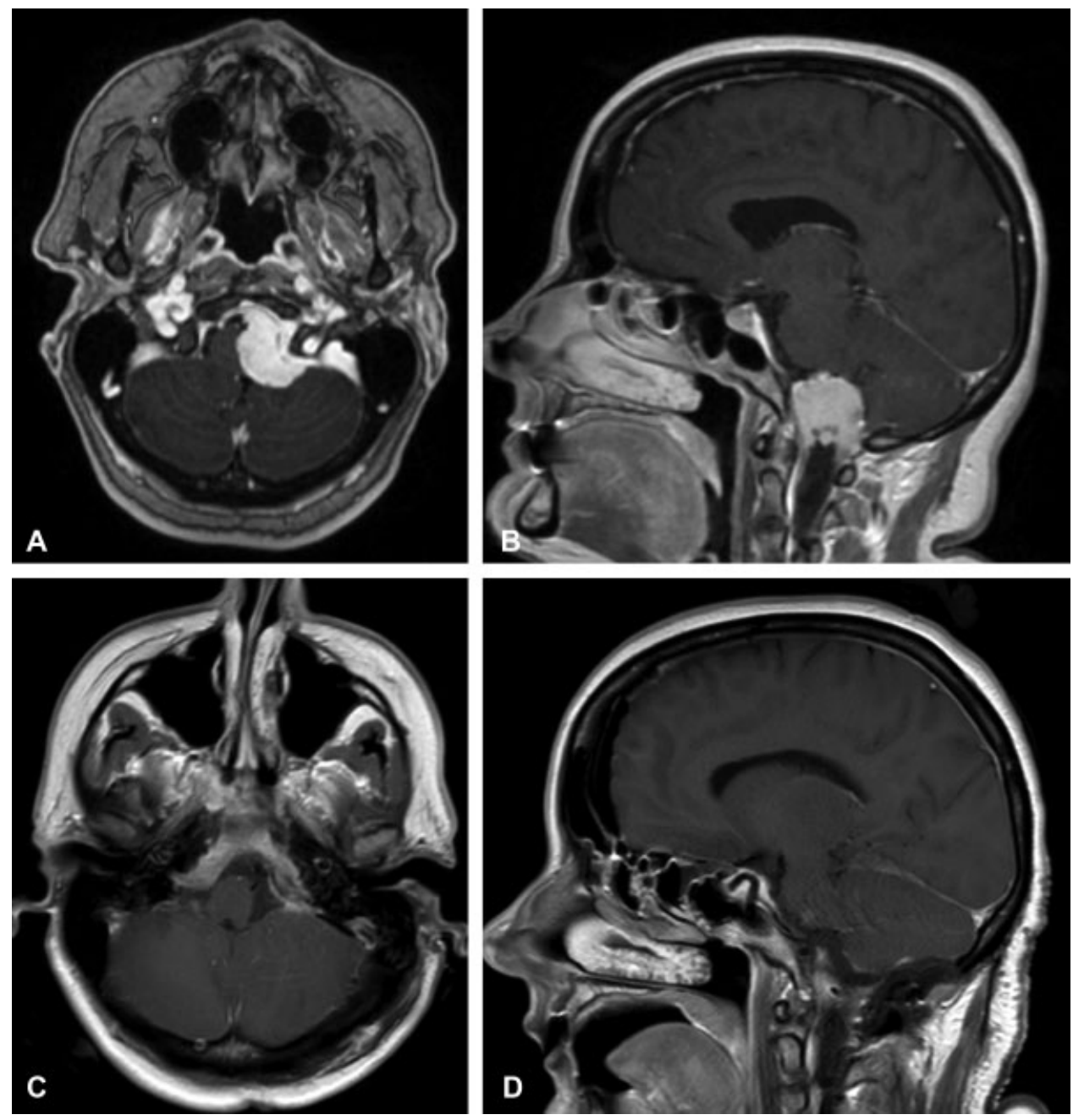

Fig. 1 T1-weighted, contrast-enhanced magnetic resonance (MR) imaging of the foramen magnum meningioma. (A) Preoperative axial image showing the foramen magnum meningioma with medullary compression. (B) Preoperative sagittal image showing the predominantly left-sided foramen magnum meningioma. (C) Postoperative axial image revealing maximum total resection of the foramen magnum meningioma. (D) Postoperative sagittal image revealing maximal total resection of the foramen magnum meningioma. (Used with permission from Barrow Neurological Institute, Phoenix, Arizona). 

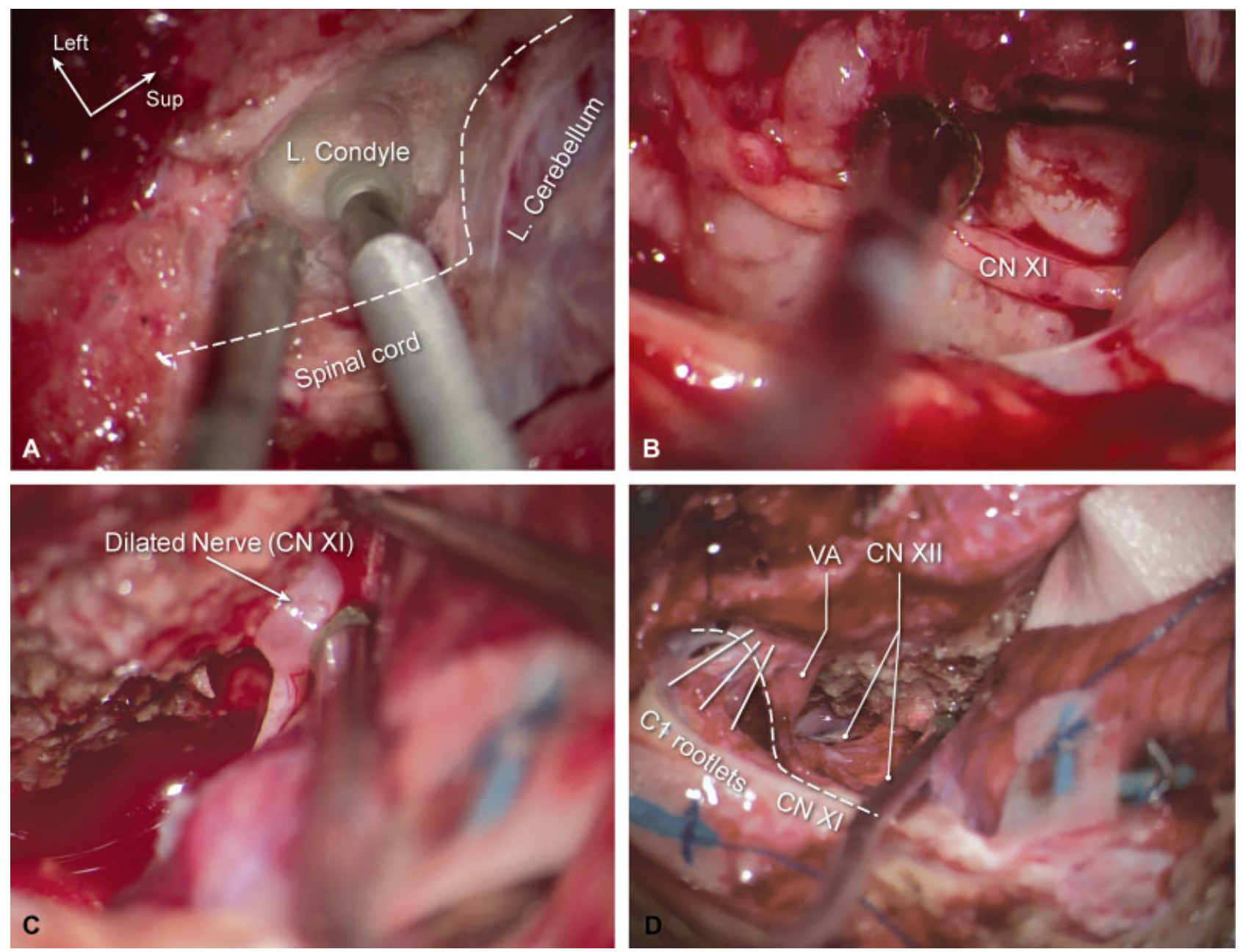

Fig. 2 Intraoperative photographs revealing key moments in the surgical approach and resection. (A) Labeled photograph displaying the key step of drilling the posterior occipital condyle. (B) Initial tumor resection after opening of the arachnoid membrane overlying the tumor. (C) A dilated nerve fascicle of CN XI noted after removal of tumor entering the jugular foramen. (D) Labeled photograph revealing tumor resection bed with preserved cranial nerves and coagulated thin tumor layer along the lateral dura. C1, cervical 1; CN, cranial nerve; VA, vertebral artery. (Used with permission from Barrow Neurological Institute, Phoenix, Arizona).

\section{Publication Comments}

This is a masterful demonstration of the "far lateral" approach to resect a left ventral foramen magnum meninigioma. This is absolutely the quintessential lesion that is best addressed with this approach. I strongly agree, there is rarely a reason, when addressing intradural pathology, to disrupt the articular surface between the occipital condyle and cervical 1 (C1). As the video demonstrates, I also often end up leaving a small plaque of tumor just ventral to the dural entry point of the ipsilateral vertebral artery. Early in my career, I would completely mobilize the vertebral artery out of the foramen transversarium of $\mathrm{C} 1$ and $\mathrm{C} 2$ to transpose it to allow access to this otherwise somewhat blind area, but I now think this is not a fruitful undertaking. It puts the artery at significant risk, takes a very long time, and by no means guarantees a surgical cure. Of note, I usually use an autologous fascia lata graft sewn in with a running 5-0 monofilament suture to get a watertight closure to avoid pseudomeningocele formation at the conclusion of tumor resection. I also prefer the "hockey-stick" incision rather than a C-shaped retroauricular incision. I feel this video will be a great primer for anyone planning to perform this approach. Congratulations to the authors on a great result.

Michael J. Link, MD Mayo Clinic, Rochester Minnesota

Disclosures

None.

Financial Support

None.

Conflict of Interest

None declared.

\section{Acknowledgments}

The authors thank the staff of Neuroscience Publications at Barrow Neurological Institute for assistance with manuscript and video preparation. 